\title{
Correction to: Participatory reporting of the 2016 bleaching event in the Western Indian Ocean
}

\author{
Mishal Gudka' ${ }^{1}$ David Obura ${ }^{1}$ James Mbugua ${ }^{1} \cdot$ Said Ahamada $^{2}$. \\ Ulli Kloiber ${ }^{3} \cdot$ Tammy Holter $^{4}$
}

Published online: 20 September 2019

(C) Springer-Verlag GmbH Germany, part of Springer Nature 2019

\section{Correction to: Coral Reefs https://doi.org/10.1007/s00338-019-01851-3}

This erratum has been initiated as authors first and last names appeared inverse in the original article and should be correctly read as:
Mishal Gudka • David Obura • James Mbugua • Said Ahamada • Ulli Kloiber • Tammy Holter

The original article has been thus corrected.

Publisher's Note Springer Nature remains neutral with regard to jurisdictional claims in published maps and institutional affiliations.

The original article can be found online at https://

doi.org/10.1007/s00338-019-01851-3.

Mishal Gudka

mgudka@cordioea.net

David Obura

dobura@cordioea.net

James Mbugua

jmbugua@cordioea.net

Said Ahamada

ahamadas@yahoo.com

Ulli Kloiber

ukloiber@gmail.com

Tammy Holter

tammy@scuba-do-zanzibar.com

1 Coastal Oceans Research and Development - Indian Ocean (CORDIO) East Africa, 9 Kibaki Flats,

P.O. Box 10135-80101, Mombasa, Kenya

2 Indian Ocean Commission, Blue Tower - 3rd floor, Ebène, Mauritius

3 Chumbe Island Coral Park Ltd, Zanzibar, Tanzania

4 Scuba Do Zanzibar, Kendwa, Zanzibar, Tanzania 